\title{
Interactions in Massive Colliding Wind Binaries
}

\author{
Michael F. Corcoran ${ }^{\dagger}$ \\ CRESST and X-ray Astrophysics Laboratory, NASA/GSFC, Greenbelt, MD 20771, USA \\ and Universities Space Research Association, 10211 Wincopin Circle, Suite 500 Columbia, MD 21044, USA
}

There are observational difficulties determining dynamical masses of binary star components in the upper HR diagram both due to the scarcity of massive binary systems and spectral and photometric contamination produced by the strong wind outflows in these systems. We discuss how variable X-ray emission in these systems produced by wind-wind collisions in massive binaries can be used to constrain the system parameters, with application to two important massive binaries, Eta Carinae and WR 140.

Keywords: stars: binaries, X-ray: stars, stars (individual): Eta Carinae, stars (individual):WR 140

\section{INTRODUCTION}

The most massive stars $(M>60 M \odot)$ play crucial roles in altering the chemical and thermodynamic properties of their host galaxies. Stellar mass is the fundamental stellar parameter that determines their ancillary properties and which ultimately determines the fate of these stars and their influence on their galactic environs. Unfortunately, stellar mass becomes observationally and theoretically less well constrained as it increases. Theory becomes uncertain mostly because very massive stars are prone to strong, variable mass loss which is difficult to model. Observational constraints are uncertain too. Massive stars are rare, and massive binary stars (needed for dynamical determination of mass) are rarer still; and of these systems only a fraction have suitably high orbital inclinations for direct photometric and spectroscopic radial-velocity analysis. Even in the small number of cases in which a high-inclination binary near the upper mass limit can be identified, rotational broadening and contamination of spectral line features from thick circumstellar material (either natal clouds or produced by strong stellar-wind driven mass loss from one or both of the stellar components) biases the analysis. In the wilds of the upper HR diagram, we're often left with indirect and circumstantial means of determining mass, a rather unsatisfactory state of affairs.

\section{WIND-WIND COLLISIONS AS A BINARY DIAG- NOSTIC}

However, in massive binaries, the strong stellar wind possessed by at least one (and, usually, both) of the stars will interact with the radiation and (in most cases) the wind outflowing from the companion star's surface. Bob Koch and his collaborators, notably Ray Pfeiffer and Ioannis Pachoulakas, recognized this fact long ago (Pfeiffer et al. 1994, Pfeiffer et al. 1997), and developed surprisingly sophisticated models of the complex radiative transfer through the "moving envelope" (to use Sobolev's terminology) of the binary: in these cases, in which the wind is moving in an azimuthal direction (due to orbital motion) in addition to the radial one.

A particularly interesting case occurs when the stellar wind from one star collides with a companion star's wind. The shock-heated gas (typically at temperatures of millions of Kelvin) produced in this collision generates intense X-ray emission that provides a useful diagnostic of wind mass-loss rates and densities. If the cooling of

(c) This is an Open Access article distributed under the terms of the Creative Commons Attribution Non-Commercial License (http://creativecommons.org/licenses/by-nc/3.0/) which permits unrestricted non-commercial use, distribution, and reproduction in any medium, provided the original work is properly cited.

Received Dec 29, 2011 Revised Jan 26, 2012 Accepted Feb 16, 2012 ${ }^{\dagger}$ Corresponding Author

E-mail: Michael.F.Corcoran@nasa.gov

Tel: +1-301-286-5576 Fax: +1-301-286-1684 
the shocked gas from the star is dominated by adiabatic expansion, then simple scaling laws show that the Xray luminosity $L_{x}$ of this gas varies inversely with stellar separation $D$, i.e. $L_{x} \propto D^{-1} \dot{M}^{2} V^{-3 / 2}(1+\Re) / \Re^{4}$ (see Stevens, Blondin \& Pollock 1992), where $\dot{M}$ is the mass loss rate, $V$ the wind speed, and $\mathfrak{R}$ the momentum flux ratio of the two winds. This change in emission, and the variable overlying wind absorption which changes with our viewing angle to the X-ray emitting gas, produces an orbital dependence which can be usefully analyzed to constrain the dynamical properties of the system, and, in the best case, constrain the mass of one or both components.

\subsection{Eta Carinae: A Test Case}

Eta Carinae $(=$ HD 93308) is a long-period $(P=2022$ days) colliding wind binary with an extremely bright unstable Luminous Blue Variable primary (Eta Car A) which has a dense $\left(\dot{M} \approx 10^{-3} \mathrm{M} \odot \mathrm{yr}^{-1}\right)$, slow $\left(V_{\infty} \approx 500 \mathrm{~km} \mathrm{~s}^{-1}\right)$ wind (Hillier et al. 2001). Eta Car A is one of the most massive and luminous stars in the Galaxy, and as such has been an object of intense interest (Davidson \& Humphreys 1997). Periodic variations in the nebular excitation and in the Xray brightness of the system (Ishibashi et al. 1999) suggest that Eta Car A is orbited by a fainter, hotter, lower mass, unseen companion (Eta Car B) possessing a less dense ( $\left.\dot{M} \approx 10^{-5} \mathrm{M} \odot \mathrm{yr}^{-1}\right)$ but much faster $\left(V_{\infty} \approx 3000 \mathrm{~km} \mathrm{~s}^{-1}\right)$ wind in a very eccentric orbit ( $e \sim 0.9$ or thereabouts). Because of the large eccentricity, changes in separation (by a factor of 20) and viewing geometry produce phase-dependent variability in nearly all bands of the electromagnetic spectrum, especially in the thermal X-ray region. This cyclical variability makes Eta Carinae a fine laboratory for studying hypersonic astrophysical shocks, the generation of high-energy thermal radiation, and (possibly) the production of non-thermal high energy emission due to Fermi acceleration of charged particles and inverseCompton scattering of seed photospheric photons (Leyder, Walter \& Rauw 2010). However, Eta Car A is also a dramatic and sporadic variable in its own right, prone to episodes of extreme brightening and mass loss (the best example of this is the "Great Eruption" of 1843, at which time the star spewed forth $\sim 10 M \bullet$ of stellar material which now forms its dusty shroud). Sporadic variations in the stellar wind from Eta Car A should cause cycle-to-cycle changes in the state of the wind-wind shock. The phasedependent and secular X-ray variations of the thermal Xray emission have been studied in great detail by the Proportional Counter Array (PCA) on board the Rossi X-ray Timing Explorer (RXTE; Bradt, Rothschild \& Swank 1993) for the last 3 stellar orbital cycles ${ }^{1}$ (from 1996 to 2011; Fig. 1 ; Corcoran et al. 2010). We define Cycle 1 to be the orbital cycle centered on the 1997 X-ray minimum, Cycle 2 centered on the 2003.5 minimum, and Cycle 3 centered on the 2009 minimum.

As discussed in Pittard \& Corcoran (2002) and Parkin et al. (2009), the X-ray variations can be modelled to yield values of the stellar wind parameters of Eta Car B, the star which has otherwise not been directly observed. Since wind terminal velocity $V$ is about 3 times the stellar escape velocity $V_{\text {esc }}$ (Abbott 1978) we can then constrain the escape velocity and effective gravity of this hidden companion. In turn, the physical parameters of the companion and the orbital elements so derived from these types of analyses can be used to constrain the stellar and massloss parameters of Eta Car A. Table 1 lists parameters derived from analysis of the $\mathrm{X}$-ray spectra and lightcurve (Pittard \& Corcoran 2002, Parkin et al. 2009).

\subsection{WR 140: A Shock Physics Laboratory}

WR 140 (= HD 193793; WC7+O4-5) is arguably the best known example of a colliding wind system, and of the range of phenomena which may be associated with

${ }^{1}$ see http://asd.gsfc.nasa.gov/Michael.Corcoran/eta_car/etacar_rxte_ lightcurve/ for the latest RXTE data

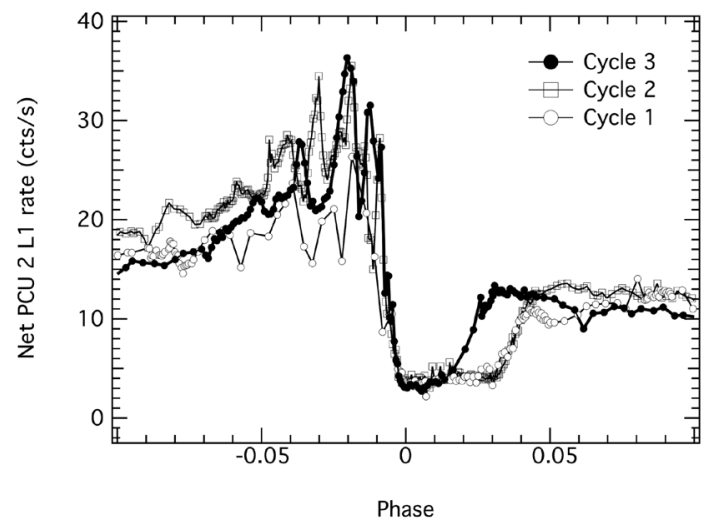

Fig. 1. RXTE PCA X-ray fluxes from Eta Car near periastron passage for 3 orbital cycles. The full lightcurve is available in Corcoran et al. (2010), with updates at http://asd.gsfc.nasa.gov/Michael.Corcoran/eta_car/etacar_ rxte_lightcurve.

Table 1. Parameters for the Eta Carinae System.

\begin{tabular}{lcc}
\hline & X-ray value & Non-X-ray value \\
\hline Mass Loss Rate $\left(M_{\odot} \mathrm{yr}^{-1}\right)$ & $2.5 \times 10^{-4} \& 10^{-5}$ & $10^{-3} \&-$ \\
Terminal Velocity $\left(\mathrm{km} \mathrm{s}^{-1}\right)$ & $500 \& 3000$ & $500 \&-$ \\
Escape Velocity $\left(\mathrm{km} \mathrm{s}^{-1}\right)$ & $200 \& 1200$ & $200 \&-$ \\
\hline
\end{tabular}


strong, time-variable astrophysical shocks. WR 140's long period $(P=2,897 \mathrm{~d}$ ) highly eccentric $(e=0.88)$ orbit (Fahed et al. 2011) is congruent to Eta Car's, but the lack of thick circumstellar material around WR 140, and the ability to detect spectral features of both stars in the system, as well as the fact that the shock has been resolved by Very Long Baseline Array interferometry (Dougherty et al. 2005) means we have a much clearer view of WR 140 and a much more direct understanding of the variations in the wind-wind interaction around the orbit.

\subsubsection{Modeling WR 140's X-ray Emission}

The PCA on RXTE has measured the X-ray lightcurve of WR 140 for over two cycles². The coverage is not as extensive or complete as it is for Eta Car, however two X-ray minima were measured in detail. Qualitatively, the phasedependent X-ray variation of WR 140 is similar to that of Eta Car: there's a gradual increase in 2-10 keV X-ray flux from apastron as the stars approach periastron passage; the X-ray flux grows as (roughly) $1 / D$ (where $D$ is the separation between the two stars) up through orbital mean anomaly $\phi \approx 0.9$, at which time a deviation from this relation begins; there's a rapid rise to a maximum flux near the time when the X-ray emitting material near the shock

${ }^{2}$ see http://asd.gsfc.nasa.gov/Michael.Corcoran/wr140/wr140_rxte_ lightcurves/ for the latest RXTE data cone apex is viewed through the lower density wind of the O4 companion; the X-ray flux falls to a minimum which occurs near the time when the leading edge of the shock cone is occulted by the WR star; and after this minimum, the flux recovers but the recovery is asymmetric, i.e. the level after the minimum is lower than the level at a similar mean anomaly prior to the minimum. Fig. 2 shows the observed 2-10 keV X-ray variation for the two periastron passages observed by RXTE.

Modeling the system with smoothed particle hydrodynamics calculations similar to those used to model the $\mathrm{X}$-ray flux variations in Eta Car has had some success. As shown in Russell et al. (2011), simple models in which the X-ray flux is localized very near the apex of the shock cone provide good descriptions of the observed variation, though models in which the X-ray emitting region is distributed along the shock cone (as expected in a realistic wind-wind collision) don't describe the observed variations as well.

\section{CONCLUSIONS}

As a canonical colliding wind binary, the X-ray flux behavior of WR 140 around the orbit is remarkably wellbehaved even if not yet entirely understood in detail. Important features of its X-ray lightcurve (X-ray extrema,

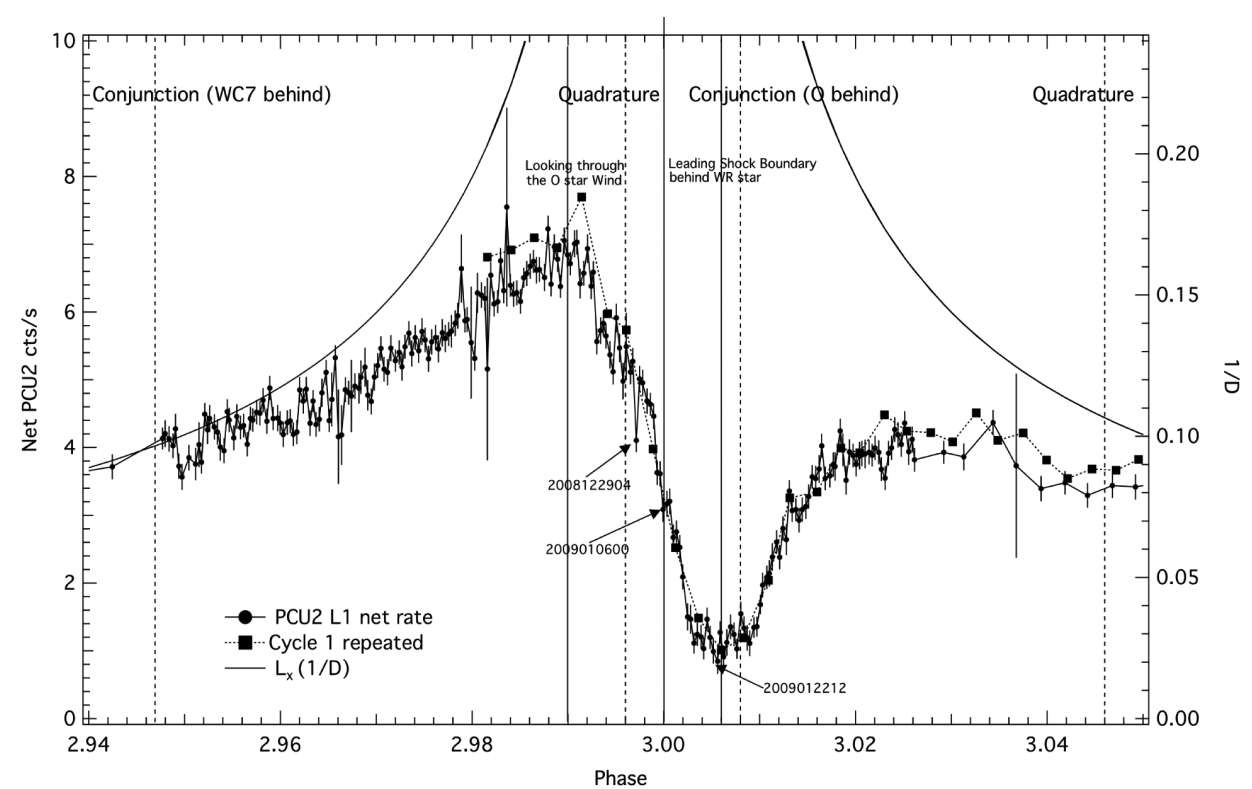

Fig. 2. RXTE PCA fluxes from WR 140 near X-ray minimum. The black circles are observations taken in 2009 , while the data marked by the square symbols are the observations of the 2001 minimum, shown for comparison. Universal Time dates of observations from 2009 are indicated, along with important orbital phases near periastron passage, which occurs at $\phi=3.00$. The solid curve shows a $1 / D$ brightness variation, expected for an adiabatic shock. 
hardness ratio maximum, etc.) are well associated with significant orbital events deduced from radial-velocity and radio interferometric studies. In the Eta Car system as well, models of the phase-locked X-ray variations have been useful in determining the system parameters. However, Eta Car is a star that's notoriously badly behaved in almost every epoch and almost every energy band, and indeed the behavior of Eta Car's X-ray flux shows significant non-phase-locked variations. Secular changes in the observed X-ray flux from Eta Car are probably the best indication of a fundamental change in the wind-wind interaction zone. Such change must indicate a real variation in the stellar winds from either or both the primary and secondary.

\section{REFERENCES}

Abbott DC, The terminal velocities of stellar winds from early-type stars, ApJ, 225, 893-901 (1978). http://dx.doi.org/ 10.1086/156554

Bradt HV, Rothschild RE, Swank JH, X-ray timing explorer mission, A\&AS, 97, 355-360 (1993).

Corcoran MF, Hamaguchi K, Pittard JM, Russell CMP, Owocki SP, et al., Recent X-ray variability of $\eta$ carinae: the quick road to recovery, ApJ, 725, 1528-1535 (2010). http:// dx.doi.org/10.1088/0004-637X/725/2/1528

Davidson K, Humphreys RM, Eta Carinae and its environment, ARA\&A, 35, 1-32 (1997). http://dx.doi.org/ 10.1146/annurev.astro.35.1.1

Dougherty SM, Beasley AJ, Claussen MJ, Zauderer BA, Bolingbroke NJ, High-resolution radio observations of the colliding-wind binary WR 140, ApJ, 623, 447-459 (2005). http://dx.doi.org/10.1086/428494

Fahed R, Moffat AFJ, Zorec J, Eversberg T, Chené AN, et al., Spectroscopy of the archetype colliding-wind binary WR 140 during the 2009 January periastron passage, MN-
RAS, 418, 2-13 (2011). http://dx.doi.org/10.1111/j.13652966.2011.19035. $\mathrm{x}$

Hillier DJ, Davidson K, Ishibashi K, Gull T, On the nature of the central source in $\eta$ Carinae, ApJ, 553, 837-860 (2001). http://dx.doi.org/10.1086/320948

Ishibashi K, Corcoran MF, Davidson K, Swank JH, Petre R, et al., Recurrent X-ray emission variations of $\eta$ Carinae and the binary hypothesis, ApJ, 524, 983-987 (1999). http:// dx.doi.org/10.1086/307859

Leyder J-C, Walter R, Rauw G, Hard X-ray identification of $\eta$ Carinae and steadiness close to periastron, A\&A, 524, A59 (2010). http://dx.doi.org/10.1051/0004-6361/ 201014316

Parkin ER, Pittard JM, Corcoran MF, Hamaguchi K, Stevens IR, 3D modelling of the colliding winds in $\eta$ Carinae-evidence for radiative inhibition, MNRAS, 394, 17581774 (2009). http://dx.doi.org/10.1111/j.1365-2966.2009. 14475.x

Pfeiffer RJ, Pachoulakis I, Koch RH, Stickland DJ, The winds of hot, close binaries. Paper 1:Y Cygni (HD 198846), Obs, 114, 297-308 (1994).

Pfeiffer RJ, Pachoulakis I, Koch RH, Stickland DJ, The winds of hot close binaries. Paper 3: HD 159176, Obs, 117, 301309 (1997).

Pittard JM, Corcoran MF, In hot pursuit of the hidden companion of eta Carinae: an X-ray determination of the wind parameters, A\&A, 383, 636-647 (2002). http:// dx.doi.org/10.1051/0004-6361:20020025

Russell CMP, Corcoran MF, Okazaki AT, Madura TI, Owocki SP, X-ray modeling of Eta Carinae \& WR140 from SPH simulations, in Proceedings of the 39th Liège Astrophysical Colloquium, eds. Rauw G, De Becker M, Naze Y, Vreux J-M, Williams P (Bulletin de la Société Royale des Sciences de Liège, Liège, Belgium, 2011), 719.

Stevens IR, Blondin JM, Pollock AMT, Colliding winds from early-type stars in binary systems, ApJ, 386, 265-287 (1992). http://dx.doi.org/10.1086/171013 